\title{
Manual method of construction: table of random two-digit numbers
}

\begin{abstract}
One manual method of construct ion of a set/table of random two-digit numbers has been thought of and the method has been applied in the construction of a set/table of random two-digit numbers. This paper describes this method and one table of random two-digit numbers that has been constructed by this method. The table constructed contains 10000 random occurrences of the 100 two-digit numbers $00,01,02,03$, ..................... 98, 99. Method of drawing of random two-digit numbers from the table has also been discussed with examples.
\end{abstract}

Keywords: two-digit numbers, table of random occurrences, drawing of random two-digit numbers
Volume 5 Issue 5 - 2017

\author{
Dhritikesh Chakrabarty,' Brajendra Kanta \\ Sarmah ${ }^{2}$ \\ 'Department of Statistics, Handique Girls' College, Gauhati \\ University, India \\ ${ }^{2}$ Department of Statistics, Bholanath College, India
}

Correspondence: Dhritikesh Chakrabarty, Department of Statistics, Handique Girls' College, Gauhati University, Guwahati-78I00I,Assam, India, Email dhritikesh.c@rediffmail.com. dhritikeshchakrabarty@gmail.com

Received: February 17, 2017 | Published: April 20, 2017

\section{Introduction}

Drawing of random sample has been found to be a vital work or basic necessity in most of the research and investigation works. The scientific method of selecting a random sample consists of the use of random number table. Several tables of random numbers have already been constructed by the renowned researchers. Some of them are (in chronological order) due to Tippett, ${ }^{1}$ Mahalanobis ${ }^{2}$ Kendall \& Smith, ${ }^{3,4}$ Fisher \& Yates, ${ }^{5}$ Hald, ${ }^{6}$ Royo \& Ferrer, ${ }^{7}$ RAND Corporation, ${ }^{8}$ Quenouille, ${ }^{9}$ Moses \& Oakford, ${ }^{10}$ Rao, Mitra \& Matthai, ${ }^{11}$ Rohlf \& Sokal, ${ }^{12}$ Manfred, ${ }^{13}$ Hill \& Hill ${ }^{14}$ etc. Among these tables, the following four tables are treated as suitable in drawing of simple random sample (with or without replacement) from a population: ${ }^{15}$

i. Tippett's Random Numbers Table that consists of 10,400 four-digit numbers giving in all 41,600 single digits selected at random from the British Census report. ${ }^{1}$

ii. Fisher and Yates Random Numbers Table that comprises 15000 digits arranged in two's. ${ }^{5}$

iii. Kendall and Smith's Random Numbers that consists of 100,000 digits grouped into 25,000 sets of random four-digit numbers. ${ }^{3}$

iv. Random Numbers Table by Rand Corporation that contains of one million digits consisting of 200,000 random numbers of 5 digits each. ${ }^{8}$

Fisher \& Yates obtained the random numbers from the $10^{\text {th }}$ to $19^{\text {th }}$ digits of A.S. Thompson's 20-figure logarithmic tables. In choosing from those digits, an element of randomness was introduced by using playing cards for the selection of half pages of the tables and of a column between $10^{\text {th }}$ to $19^{\text {th }}$ and finally for allotting these digits to the 50th place in a block. In this case, the question arises whether the method applied in selecting the numbers has made the numbers random. Recently, some studies have been made on the testing of randomness of the table due to Fisher \& Yates $^{5}$ where it has been found that this table, consisting of the 7500 occurrences of the 100 two-digit numbers, is not properly random and deviates significantly from proper randomness [Chakrabarty, ${ }^{16}$ Sarmah \& Chakrabarty. ${ }^{17}$ ] Thus, there is necessity of constructing of a table of random twodigit numbers. One manual method of construction of a set/table of random two-digit numbers has been thought of and the method has been applied in the construction of a set/table of random two-digit numbers. This paper describes this method and one table of random two-digit numbers that has been constructed by this method. The table constructed contains 10000 random occurrences of the 100 two-digit numbers $00,01,02,03$, , 98, 99 .

Method of drawing of random two-digit numbers from the table has also been discussed with examples.

It is to be noted that there is available provision of accessing to computers for generating random numbers. However, the random numbers generated by computer are not properly random but pseudo random since the numbers are generated by some pre-fixed rule.

\section{Construction of set of random two-digit numbers}

Let us mention the two definitions of probability namely the definition of probability in theoretically ideal situation $\left(\right.$ Chakrabarty $\left.{ }^{18}\right)$ and definition of probability in practically ideal situation (Chakrabarty ${ }^{19}$.)

\section{Definition of probability on theoretically ideal situation}

First definition: If, in the theoretically ideal situation, a trial is repeated sufficient times and if an $E$ occurs $n m$ times out of $n M$ repetitions of the trial (for $n=1,2,3, \ldots \ldots \ldots$ ) then the probability of occurrence of the event denoted by $P(E)$, can be defined by

$$
p(E)=\frac{m}{M}
$$

where $m$ is the number of times the $E$ occurs out of $M$ repetitions of the trial. 
Second definition: The probability of an event $E$, denoted by $p(E)$, associated to a random experiment is a rational number $\frac{m}{M}$ such that if, in the theoretically ideal situation, the associated trial is repeated sufficient times, the event $E$ occurs $n m$ times out of $n M$ repetitions of the trial $(n=1,2,3, \ldots \ldots \ldots)$.

Remark: These two definitions imply that if $E$ is an event associated to a random experiment then

$$
p(E)=\frac{m}{M}
$$

if and only if $E$ occurs $n m$ times out of $n M$ repetitions of the trial (for $n=1,2,3, \ldots \ldots \ldots$ ) in the theoretically ideal situation.

\section{Definition of probability on practically ideal situation}

Third definition : If, in the practically ideal situation, a trial is repeated sufficient times and if more than $100(1-\alpha) \%$ cases of the fact that an event $\mathrm{E}$ occurs $\mathrm{nm}$ times out of $n M$ repetitions of the trial (for $n=1,2,3, \ldots \ldots \ldots$ ) will be true and /or if the number of occurrence of $E$ lies between $(\mathrm{nm}-\alpha \%$ of $n m) \&(n m+\alpha \%$ of $n m)$ out of nM repetitions of the trial (for $n=1,2,3, \ldots \ldots \ldots$ ) then the probability of occurrence of the event denoted by $p(E)$, can be defined

$$
p(E)=\frac{m}{M}
$$

Fourth definition: The probability of an event E, denoted by $p(E)$ , associated to a random experiment is a rational number $\frac{m}{M}$ such that if, in the practically ideal situation, the associated trial is repeated sufficient times then more than $100(1-\alpha) \%$ cases of the fact that an event $E$ occurs nm times out of $n M$ repetitions of the trial (for $n=1,2,3, \ldots \ldots \ldots)$ will be true and/ or if the number of occurrence of $E$ lies between $(n m-\alpha \%$ of nm) \& (nm $+\alpha \%$ of nm) out of $n M$ repetitions of the trial (for $\mathrm{n}=1,2,3, \ldots \ldots \ldots$ )

Remark: These two definitions imply that if $E$ is an event associated to a random experiment then

$$
p(E)=\frac{m}{M}
$$

if and only if more than $100(1-\alpha) \%$ cases of the fact that the event $E$ occurs $n m$ times out of $n M$ repetitions of the trial (for $n=1,2,3, \ldots \ldots . .$.$) will be true and/or if the number of occurrence$ of $E$ lies between $(n m-\alpha \%$ of $n m)$ times \& $(n m+\alpha \%$ of $n m)$ times out of $n M$ repetitions of the trial (for $n=1,2,3, \ldots \ldots \ldots$ ).

Performing of an experiment has here been termed as a theoretically ideal experiment if it does not suffer from any error (even the chance error too). The corresponding situation under which an experiment is performed has here been termed as theoretically ideal situation. Similarly, performing of an experiment has here been termed as a practically ideal experiment if it does not suffer from any other error except from possible chance error whose probability is not more than $\alpha$. The corresponding situation under which an experiment is performed has here been termed as practically ideal situation. Here $\alpha$ is the fraction ( $\alpha \%$ is the percentage), which is as per one's own choice, of the deviation from proper randomness." $\alpha$ is termed here as acceptable level of chance error (abbreviated as ALOCE).

The idea or the concept of probability is the basis of describing/ explaining the randomness of an event and hence of a set of numbers.
The philosophy behind the definitions of probability in theoretically ideal situation and in practically ideal situation can be applied in the construction of a random number table.

The random numbers' set constructed here are based on this philosophy. The concept of randomness of the occurrences of the 100 two-digit numbers can be explained/defined as follows:

Consider the occurrences, in the following set of the two letters namely $\mathrm{A} \& \mathrm{~B}$ in the following order:

$\{B, A, B, B, A, B, A, B, A, A, B, B, B, A, B, A, A, B, A,, A$,

The occurrences of A \& B can be termed as random if the probabilities of their occurrences in the set are equal.

By the same logic, one can define the randomness of the set of 100 two-digit numbers

00, 01, 02, 03, 04, 05, 06, 07, 08, 09, 10,11, 12,13,14,15, 16, 17, 18, 19, 20, 21, 22, 23, 24, $25,26,27,28,29,30,31,32,33,34,35,36,37,38,39,40,41,42,43,44,45,46,47,48,49$, $50,51,52,53,54,55,56,57,58,59,60,61,62,63,64,65,66,67,68,69,70,71,72,73,74$, $75,76,77,78,79,80,81,82,83,84,85,86,87,88,89,91,91,92,93,94,95,96,97,98,99$

Suppose that a trial of drawing of a number from among the 100 two-digit numbers has been performed many times and a set/table of the drawn numbers has been constructed placing them in the order of their occurrences. The set/table will then be random if all the numbers have equal chance (and hence equal probability which is 0.01 ) of occurring in the set/table.

Conversely, a set/table consisting of a total of $N$ occurrences of the 100 two-digit numbers will be random if and only if all the 100 two-digit numbers have equal chance (and hence equal probability which is 0.01 ) of occurrence in the set/table. Consequently, as per the second definition the set/table will be random if and only if each of the 100 two-digit numbers occurs $n$ times out of $100 n$ trials ( $n=1,2, \ldots \ldots \ldots)$ in theoretically ideal situation and as per the fourth definition the set/table can be treated as random at ALOCE $\alpha$ if and only if the number of occurrence of each of them lies between $(n-\alpha \%$ of $n) \&(n+\alpha \%$ of $n)$ in practically ideal situation.

The randomness of a set/table of two-digit numbers and of a set/ table of three-digit numbers can be defined in a similar manner.

\section{Method of construction}

While constructing a table of random two-digit numbers one is required to note that the table will be random at ALOCE $\alpha$ if and only the number of occurrence of each of the 100 two-digit numbers, out of $100 n$ trials $(n=1,2, \ldots \ldots \ldots)$, lies between $(n+\alpha \%$ ofn $)$ and $(n-\alpha \%$ ofn $)$ as per the above two definitions of probability.

Let us take an opaque container and 100 small identical balls identifying them by the 100 two-digit numbers as mentioned above. The container to be taken is to be opaque in order to make the experimenter blinded so that unbiasness of the trials to be performed can be achieved. Let the 100 balls be put inside the container and make the balls well shuffled.

If the 100 balls are drawn one by one, by applying the principle of blinding, from the container and the numbers appeared on the balls are listed in the order of their occurrences then 100 observations will be obtained where each of the 100 two-digit numbers will appear once. 
If the process is repeated and the observations obtained are combined with the earlier ones then 200 observations will be obtained where each of the 100 two-digit numbers will appear twice.

If the process is continued, $100 n$ observations will be obtained where each of the 100 two-digit numbers will appear $n$ times out of $n$ repetitions $(n=1,2,3,4, \ldots \ldots \ldots)$.

If a set/table of $100 \mathrm{~N}$ numbers is constructed by this process then out of any $100 n$ consecutive occurrences of the two-digit numbers the number of occurrence of each of the 100 two-digit numbers lies between $n \pm 1$. Therefore, the set/table of the observations obtained above will be a random numbers' set/table of the 100 two-digit numbers at ALOCE 0.01

By this method, one sets/tables each containing 10000 random occurrences of the 100 two-digit have been constructed which have been shown in (Table 1).

\section{Method of drawing of random two-digit numbers from the table}

Following are some prime characteristics of the set/table of random two-digit numbers constructed here

i. In the table, each of the 100 two-digit numbers occurs $n$ times out of $100 n$ consecutive occurrences $(n=1,2, \ldots \ldots$.$) if we$ start counting from the observation at the $(100 k+1)^{\text {th }}$ position ( $k=0,1,2,3, \ldots \ldots \ldots \ldots . . .$.

ii. In the table, the frequency of occurrence of each of the 100 two-digit numbers out of $100 n$ consecutive trials $(n=1,2,3, \ldots \ldots \ldots$ ) may be one more or less than $n$ if we start counting from any position.

iii. The table can be treated as random as per the philosophy behind the two definitions of probability namely definition in theoretically ideal situation [given by the First Definition \& the Second Definition] and definition in practically ideal situation [given by the Third Definition \& the Fourth Definition].

iv. The table is random with respect to the occurrences of the numbers row-wise but not column-wise. Thus while drawing random numbers from the table, one requires moving row-wise either to the right or to the left starting from any position in the table. The starting position and the direction of movement are to be selected at random by suitable randomized trials in order to keep their randomness intact.

The table, constructed here, can be used in drawing of random two-digit numbers

i. which are distinct

ii. which are not necessarily distinct.

\section{Drawing of distinct random two-digit numbers}

Suppose that we want to draw $n$ random two-digit numbers from the table such that the drawn numbers are distinct.

Since distinct two-digit numbers are to be drawn, one can draw a maximum of 100 such numbers since the total number of such numbers is 100 .
As per the Characteristic No ii. mentioned above, if $n$ two-digit numbers occurred consecutively from the $(100 k+1)^{\text {th }}$ position ( $k=0,1,2, \ldots \ldots \ldots)$ in the table are drawn subject to the Characteristic No iv then the drawn $n$ numbers will be distinct and random.

Also, Characteristic No iii mentioned above implies that if $n$ twodigit numbers occurred consecutively in the table are drawn starting from any position then the drawn $n$ numbers may not be distinct ; some of them may occur twice.

Thus in order to draw distinct numbers, it is required to exclude the next occurrence of the same number and to draw the next consecutive number occurred in the table following the Characteristic No iv.

Thus the drawing of random two-digit numbers consists of the two basic tasks namely

i. selection of the starting position at random

ii. Selection of the direction (right or left) of movement at random.

Accordingly, in order to obtain the $\boldsymbol{n}$ random two-digit numbers one is to proceed with the following steps

i. Select the position, from where to start, at random. Since the table contains 10000 random occurrences of the 100 two-digit numbers, accordingly there are 10000 positions of the numbers namely 0000, 0001, 0002, 9998, 9999

In selecting the starting position, one thus can apply some usual manual randomization technique of drawing one number from among the numbers $0000,0001,0002$, ,9998, 9999.

One method of drawing of such number is as follows:

Take a set of 10 identical small balls distinguishing them by marking with the 10 digits

$0,1,2,3,4,5,6,7,8,9$ and put them inside a opaque container, say $C_{1}$

Similarly, take another set of 4 identical small distinguishing them by marking $L, R, M_{1}, M_{2}$ respectively and another opaque container, say $C_{2}$.

Now, draw one ball at random from the container $C_{1}$ containing the 10 balls and note down digit appeared on it. Let the digit is $d_{1}$.

Next, draw another ball at random from the container $C_{1}$ containing the same 10 balls and note down digit appeared on it. Let the digit is $d_{1}$.

Next, draw another ball at random from the container $C_{1}$ containing the same 10 balls and note down the digit appeared on it. Let the digit is $d_{2}$.

Then, draw one ball at random from the container $C_{2}$ putting 2 balls marked with L \& R inside it.

If the drawn ball is $\mathrm{R}$ then put the digit $d_{2}$ at the right position of $d_{1}$ and if the drawn ball is $\mathrm{L}$ then put the $\operatorname{digit}^{2} d_{2}$ at the left position of $d_{1}^{1}$.

Thus if the ball $\mathrm{R}$ appears then the selected two-digit number will be $d_{1} d$ and if the ball L appears then the selected two-digit number will be $d_{2}^{2} d_{1}$. 
Let the selected two-digit number be $d_{2} d_{1}$.

Next, draw another ball at random from the container $C_{1}$ containing all the 10 balls and note down the digit appeared on it. Let the digit be $d_{3}$.

Then, draw one ball at random from the container $C_{2}$ putting 3 balls marked with $L, M_{1} \& R$ inside it and put the digit $d_{3}$ at the left position of $d_{2} d_{1}$ if the drawn ball is $\mathrm{L}$, the middle position of $d_{2} d_{1}$ if the drawn ball is $\mathrm{M}_{1} \&$ the right position of $d_{2} d_{1}$ if the drawn ball is $R$.

Thus the selected three-digit number will be $d_{3} d_{2} d_{1}$ or $d_{2} d_{3} d_{1}$ or $d_{2} d_{1} d_{3}$ in accordance with the selected ball is $L, M_{1}$ or $R$.

Let the selected three-digit number be $d_{2} d_{3} d_{1}$.

Finally, draw another ball at random from the container $C_{1}$ containing all the 10 balls and note down the

digit appeared on it. Let the digit is $d_{4}$.

Then, draw one ball at random from the container $C_{2}$ putting 4 balls marked with $\mathrm{L}, \mathrm{M}_{1}, \mathrm{M}_{2} \& \mathrm{R}$ inside it and put the digit $d_{4}$ at the left position of $d_{2} d_{3} d_{1}$ if the drawn ball is $L$, the $1^{\text {st }}$ middle position (from left) of $d_{2} d_{3} d_{1}$ if the drawn ball is $M_{1}$, the $2^{\text {nd }}$ middle position (from left) of $d_{2} d_{3} d_{1}$ if the drawn ball is $M_{2} \&$ the right position of $d_{2} d_{3} d_{1}$ if the drawn ball is $R$.

Thus the selected four-digit number will be $d_{4} d_{3} d_{2} d_{1}$ or $d_{2} d_{4} d_{3} d_{1}$ or $d_{2} d_{1} d_{4} d_{3}$ or $d_{2} d_{1} d_{3} d_{4}$ in accordance with the selected ball is $\mathrm{L}$ or $\mathrm{M}_{1}$ or $\mathrm{M}_{2}$ or $\mathrm{R}$.

This selected number will be the required starting position.

ii. Let the $i^{\text {th }}$ position be selected as the starting position. Draw the number that occurs at the $i^{\text {th }}$ position in the table.

Chose whether to move towards left or towards right. The choice can be made at random by performing a random binary trial (for example, by tossing of an unbiased coin or by drawing a number from the container $C_{2}$ putting two identical balls, marked with $L$ and $R$ respectively, inside it.

iii. If it is chosen to move towards right, draw the numbers occurred at the positions

$$
i, i+1, i+2, \ldots \ldots \ldots \ldots . ., i+n+1
$$

in the table to obtain the $n$ random two-digit numbers.

iv. If it is chosen to move towards left, draw the numbers occurred at the positions

$i, i-1, i-2, \ldots \ldots \ldots \ldots . ., i-n+1$

in the table to obtain the $n$ random two-digit numbers.

v. It may occur that some number or numbers among those drawn may be occurred twice. In that situation, retain only one occurrence of them and draw additional numbers appeared at the consecutive positions in the table as per requirement. If $k$ additional numbers are required to draw, then draw the numbers occurred at the positions

$$
i+n, i+n+1, i+n+2, \ldots \ldots \ldots ., i+n+k-1
$$

if it is chosen to move towards right and draw the numbers occurred at the positions

$$
i-n, i-n-1, i-n-2, \ldots \ldots \ldots . ., i-n-k+1
$$

if it is chosen to move towards left.

\section{Drawing of random two-digit numbers (not necessarily distinct)}

Characteristic No i and Characteristic No ii, mentioned above, imply that if two-digit numbers are picked up at a gap of $g$ positions $(101<\mathrm{g}<199)$, the picked up numbers will not necessarily be distinct.

Thus in order to draw $n$ random two-digit numbers which need not necessarily be distinct, one is to proceed with the following steps:

i. Select one position from where to start at random by the similar method as in the case of drawing of distinct random two-digit numbers mentioned above. Let the $i^{\text {th }}$ position be selected.

ii. Draw the number that occurs at the $i^{\text {th }}$ position in the table.

iii. Chose the length of jump that is to be 101 or more and 199 or less at random. It can be chosen by some usual manual randomization technique of drawing one number from among the numbers 101, 102,103, , 198, 199.

Let the selected length of jump be $l$.

The random selection of the length of the jump can be done by similar method as done in the selection of the starting position.

iv. Chose whether to jump towards left or towards right. The choice can be made by the same method as in the earlier case.

v. If it is chosen to jump towards right, draw the numbers occurred at the positions

$$
i, i+l, i+2 l, \ldots \ldots \ldots \ldots . ., i+(n-1) l
$$

in the table to obtain the required $n$ random two-digit numbers.

v. If it is chosen to move towards left, draw the numbers occurred at the positions

$$
i, i-l, i-2 l, \ldots \ldots \ldots \ldots . ., i-(n-1) l
$$

in the table to obtain the required $n$ random two-digit numbers.

\section{Example}

\section{Example: drawing of distinct random two-digit numbers}

Let it be wanted to draw 10 distinct random two-digit numbers from (Table 1).

Suppose that the starting position selected at random be 4916 . The two-digit number at this position in (Table 1) is 52 . Thus this is selected as the $1^{\text {st }}$ one among the tens.

Suppose that it is chosen by random trial to move towards the right direction. Then the numbers at the positions 4915, 4914, 4913, 4912, 4911, 4910, 4909, 4908, 4907, are to be drawn.Now the two-digit numbers at the next 9 positions in Table 1 are 76, 90, 37, 63, 12, 74, $21,54,11$. 
Therefore, the 10 distinct random two-digit numbers will be 52 , $76,90,37,63,12,74,21,54,11$.

\section{Example: drawing of distinct random two-digit numbers}

Let it be wanted to draw 10 distinct random distinct two-digit numbers from (Table 1).

Suppose that the starting position selected at random be 9994 . The two-digit number at this position in (Table 1) is 61 .

Thus this is selected as the $1^{\text {st }}$ one among the 20 numbers to be selected.

Suppose that it is chosen by random trial to move towards the right direction. Then the numbers at the next 9 successive positions are to be selected. However, after the next 5 positions, the table comes to the end. The remaining 4 positions are then taken from the beginning of the table treating the table to be a circular one. Then the numbers at the positions 9995, 9996, 9997, 9998, 9999, 0000, 0001, 0002, 0003 are to be drawn. The two-digit numbers at the these positions in (Table 1) are $19,21,34,40,92,26,31,27,78$ respectively.

Accordingly, the 10 distinct random two-digit numbers drawn from (Table 1) are 61, 19, 21, 34, 40, 92, 26, 31, 27, 78 .

\section{Example: drawing of random two-digit numbers (not necessarily distinct):}

Let it be wanted to draw 20 random two-digit numbers from (Table 1) which are not necessarily be distinct.

Suppose that the starting position selected at random be 4324 . The two-digit number at this position in (Table 1) is 43 . Thus this is selected as the $1^{\text {st }}$ one among the 20 numbers to be selected.

Suppose that it is chosen by random trial to move towards the right direction.

Let the length of jump selected at random be 110. Then the next 19 positions in (Table 1) to be considered (treating the table as circular) will be 4434, 4544, 4654, 4764, 4874, 4984, 5094, 5204, 5314, 5424, 5534, 5644, 5754, 5864, 5974, 6084, 6194, 6304, 6414.

The number appeared at these positions in (Table 1) are 03, 17, $27,53,34,55,01,58,04.05,49,48,04,31,10,97,27,42,05$ respectively.

Accordingly, the 20 random two-digit numbers drawn from (Table 1) are $43,03,17,27,53,34,55,01,58,04.05,49,48,04,31,10$, $97,27,42,05$.

\section{Conclusion}

The method of construction of set/table of random two-digit numbers described here is purely a manual one.

The table of two-digit numbers constructed here can be treated as random as per the philosophy behind the two definitions of probability namely definition in theoretically ideal situation [given by the First Definition \& the Second Definition] and definition in practically ideal situation [given by the Third Definition \& the Fourth Definition].

The table constructed here can be applied in drawing of distinct random two-digit numbers and also in drawing of random two-digit numbers which need not necessarily be distinct.
One can think of the possibility of drawing of random four-digit numbers, random six-digit numbers etc. from this table. There is scopes of further research in this aspect.

By applying the method, which is purely a manual, one can construct larger set/table of random two-digit numbers if necessary.

Similar method can be developed for construction of set/table of random three-digit numbers and of other-digit numbers. ${ }^{20}$

\section{Constructed random numbers' table: (Table 1).}

10000 occurrences of random two-digit numbers.

\section{Acknowledgement}

None.

\section{Conflict of interest}

None.

\section{References}

1. Tippett LHC (1927) Random Sampling Numbers, Tracts for Computers no. 15, Cambridge University Press, Cambridge, England.

2. Mahalanobis PC (1934) Tables of random samples from a normal population. Sankya 1: 289-328.

3. Kendall MG, Smith BB (1938) Randomness and Random Sampling Numbers.Jour. Roy. Stat. Soc 101(1): 147-166.

4. Kendall MG, Smith BB (1939) A Table of Random Sampling Numbers", Tracts for Computers no. 24, Cambridge University Press, Cambridge, England.

5. Fisher RA (1938) Statistical Tables for Biological, Agricultural and Medical Research $6^{\text {th }}$ Edition (1982), Longman Group Limited, England 37-38 \& 134-139.

6. Hald A (1952) Table of random numbers, In A. Hald Statistical Tables and Formulas. Wiley.

7. Royo J, Ferrer S (1954) Tables of Random Numbers Obtained from Numbers in the Spanish National Lottery. Trabajos de Estadistica 5:247256.

8. Rand Corporation (1955) A Million Random Digits with 100,000 Normal Deviates, Free Press, Glencoe, Illinois.

9. Quenouille MH (1959) Tables of Random Observations from Standard Distributions. Biometrika 46: 178-202.

10. Moses EL, Oakford VR (1963) Tables of Random Permutations. George Allen \& Unwin.

11. Rao CR, Mitra SK, Matthai A(1966) Random Numbers and Permutations, Statistical Publishing Society, Calcutta,India.

12. Rohlf FJ, Sokal RR (1969) Ten Thousand Random Digits, In Rohlf \& Sokal Statistical Tables, Freeman.

13. Manfred Mohr (1971) Le Petit Livre de Nombres au Hasar. Édition d'artiste, Paris.

14. Hill ID, Hill PA (1977) Tables of Random Times. UK.

15. Cochran (1940) A Survey of Experimental design, Mimeo, U.S.D.A.

16. Chakrabarty Dhritikesh (2010) Chakrabarty's Definition of Probability Proper Randomness of Fisher and Yates Random Number Table, Int. J. Agricult. Stat. Sci 6(2): 461-469. 
17. Sarmah Brajendra Kanta, Chakrabarty Dhritikesh (2015) Testing of Randomness of the Numbers Generated by Fisher and Yates ", AryaBhatta J. MathInfo. 7(1): 87-90.

18. Chakrabarty Dhritikesh (2004) A Theoretical Definition of Probability Based on Common Sense, Bulletin of Pure and Applied Sciences - E 23E(2): 343-349.
19. Chakrabarty Dhritikesh (2011) Probability in Ideal Situation and in Practical Situation. Arya Bhatta J. Math. \& Info 3(1): 161-168.

20. Snedecor GW, Cochran WG (1967) "Statistical Methods", Iowa State University Press, Ames, Iowa, $6^{\text {th }}$ Edition. 